\title{
Decentralized Model Predictive Control of Plug-in Electric Vehicles Charging based on the Alternating Direction Method of Multipliers
}

\author{
Roberto Germanà*, Francesco Liberati, Alessandro Di Giorgio
}

\begin{abstract}
This paper presents a decentralized Model Predictive Control (MPC) for Plug-in Electric Vehicles (PEVs) charging, in presence of both network and drivers' requirements. The open loop optimal control problem at the basis of MPC is modeled as a consesus with regularization optimization problem and solved by means of the decentralized Alternating Direction Method of Multipliers (ADMM). Simulations performed on a realistic test case show the potential of the proposed control approach and allow to provide a preliminary evaluation of the compatibility between the required computational effort and the application in real time charging control system.
\end{abstract}

\begin{tabular}{cl}
\multicolumn{1}{c}{ NOMENCLATURE } \\
$t$ & Current time \\
$t_{r}^{d e p}$ & Departure time of vehicle $r \in R_{t}$ \\
$T_{t}$ & Set of time instants in the control window \\
$R_{t}$ & Set of charging PEVs at time $t$ \\
$p$ & PEV charging power \\
$S O C$ & PEV state of charge \\
$S O C^{r e f}$ & Desired PEV state of charge \\
$e$ & PEV state of charge error \\
$\xi$ & PEV conversion losses \\
$P$ & Scheduled aggregated charging power \\
$P^{r e f}$ & Reference aggregated charging power \\
$E$ & Aggregated charging power tracking error \\
$D$ & PEV cost function \\
$C$ & Load area cost function \\
$\Delta t$ & Sampling time \\
$r, \tau$ & Generic elements of sets $R_{t}, T_{t}$ (subscript) \\
$\rho$ & augmented lagrangian parameter \\
$\epsilon$ & ADMM tolerance
\end{tabular}

\section{INTRODUCTION}

Following the achievements of the 2020 climate and energy framework [1], the European Commission has established new challenging targets for 2030, with the objective of gradually decarbonizing the energy sector by moving away from fossil fuels [2]. In this context a key role is going to be played by plug-in electric vehicles (PEVs), provided that the aggregated power needed for charging is controlled to match power generation from renewables and keep feasible operation of electricity distribution grids [3], [4]. The problem of PEV charging control has been intensively investigated

* corresponding author

This work has been carried out in the framework of the 5G-SOLUTIONS project, which has received funding from the European Union's Horizon 2020 research and innovation programme under grant agreement No 856691. The content of this paper reflects only the author's view; the EU Commission/Agency is not responsible for any use that may be made of the information it contains.

R. Germanà, F. Liberati and A. Di Giorgio are with the Department of Computer, Control, and Management Engineering Antonio Ruberti at Sapienza University of Rome, Via Ariosto, 25, 00185 Rome, Italy, e-mail: \{germana,liberati, digiorgio\}@diag.uniroma1.it. during the last decade: beyond the satisfaction of driver preferences for charging, typical requirements taken into account at network level involve voltage regulation [5][7], active power regulation [8], [9], minimization of power losses [10], [11], overload avoidance [12]-[14], balancing among charging power and local generation [15]-[17], etc. Several methodologies have been applied such as several forms of optimization [18], [19], learning techniques [20], model predictive control (MPC) [21]-[24], etc.; among them MPC has been successfully field tested in the context of some EU research projects [25] and has been applied in pioneer production environments [26]. However the need of solving in real time the MPC inherent optimization problem raises concerns, especially when increasing the number and duration of simultaneous charging sessions. Though complexity can be partially broken by clustering charging PEVs according to the electricity grid topology [27] and substations power rating, promising results are expected to come from the machinery of well established decentralized optimization techniques [28], using which a set of less challenging problems are solved in parallel and driven iteratively towards the optimal solution of the original centralized problem.

In this paper we propose an original application of the alternating direction method of multipliers (ADMM) to the problem of decentralized PEVs charging control, in presence of requirements concerning the regulation of the aggregated PEVs charging power and the respect of drivers' privacy [29] and charging preferences; we model the optimization problem at the basis of the MPC framework as a consensus with regularization optimization problem and we take advantage of ADMM in order to achieve an effective real time decentralized control implementable in practice in realistic charging scenarios. The proposed formulation reconsiders the control objectives characterizing the successfully field tested centralized formulation presented in [30] and [31], also taking into account some decentralized optimization concepts characterizing the preliminary work [32].

This work raises in the context of the EU research project 5G Solutions [33], which aims to show the effectiveness of the $5 \mathrm{G}$ communication technology in use cases defined over several application fields: in the context of smart grids, its low latency and high reliability are key features expected to allow real time decentralized PEVs charging control techniques characterized by a potentially large number of communications among the optimization agents.

The remainder of the paper is organized as follows. Section II outlines the charging scenario and the control objectives. Section III presents the problem formulation. Section 
IV at first recalls the main concepts and results at the basis of ADMM and consensus with regularization optimization problem, and then shows how the proposed formulation can be put in this latter form. Section V is dedicated to the presentation of simulations and the discussion of the related results. In section VI the concluding remarks are drawn together with the expected future developments.

\section{Scenario And Control Objectives}

The reference scenario considers a load area, namely a node of the electricity distribution grid hosting a set of charging stations available for the provisioning of the charging service; all the charging stations share the same point of connection to the grid (typically a MV/LV substation). A reference charging power is assigned to the load area, representing a target load curve optimizing some operation criteria; this reference may be in principle time-dependent and subject to modifications over the time through shortterm notification (demand side management (DSM) signal). Each driver connecting his/her PEV to a charging station is expected to declare the initial and desired state of charge (SOC) and the time of departure from the charging station.

The control system is in charge of computing in real time the charging power setpoints that each PEV has to actuate during its charging session; in this work we consider the following control objectives:

1) Power tracking. The aggregated charging power in the load area has to track the time-dependent reference.

2) Charging preferences. Each PEV has to reach the desired SOC level no later than the departure time.

3) Privacy. The charging power setpoint has to be computed locally (i.e. at the level of each PEV) without sharing PEV parameters and driver's charging preferences with external agents.

For a detailed explanation of the reference scenario and related use cases the reader can refer to the previous work [30].

\section{Control Problem Formalization}

The proposed control is based on the well known MPC methodology [34]. At each sampling time a measure (or at least an estimation) of the SOC is taken from all connected PEVs; then, a proper open loop optimal control problem is solved, which provides an optimal control sequence over the control window for each PEV, whose the first sample is actually applied to the plant. The procedure is then repeated at the upcoming sampling time to realize a real time closed loop control framework.

In the following we define the open loop optimal control problem at the basis of the MPC framework. In what follows $t$ denotes the current time, $T_{t}=\{t, t+1, \ldots, t+H-1\}$ is the set of discrete time instants within the control window starting from $\mathrm{t}, \tau$ the generic time instant within the set. The set $R_{t}$ is introduced to denote the PEVs connected to the charging stations at $t$ and consequently being part of the control problem.

The open loop optimal control problem is built taking into account the requirements reported in Section II, together with technical limitations and constraints related both to the PEVs and the load area. In order to model requirements 1 and 2 we consider a target function of the form

$$
J=\sum_{\tau \in T_{t}}\left[\sum_{r \in R_{t}} D_{r}\left(e_{r}(\tau / t)\right)+C(E(\tau / t))\right]
$$

in which $D_{r}$ and $C$ are proper functions accounting for performances at PEVs and load area levels respectively. As far as concerns each PEV, a function $D_{r}$ is introduced to model the disutility resulting from the deviation of the current state of charge from the desired one. Defining the SOC error at $\tau$ as $e_{r}(\tau / t)=S O C_{r}(\tau / t)-S O C_{r}^{r e f}$, the function $D_{r}$ is taken as

$$
D_{r}\left(e_{r}(\tau / t)\right)=\alpha_{r} e_{r}^{2}(\tau / t) \quad \forall \tau \in T_{t}
$$

where $\alpha_{r}$ represents a weight used to balance the charging preference requirements among different agents.

As far as concerns the load area, the function $C$ is introduced to penalize the deviation of the aggregated charging power with respect to the reference power. Defining the tracking error at $\tau$ as $E(\tau / t)=P(\tau / t)-P^{r e f}(\tau / t)$, the function $\mathrm{C}$ is taken as

$$
C(E(\tau / t))=\beta(\tau / t) E(\tau / t)^{2} \quad \forall \tau \in T_{t}
$$

where $\beta(\tau / t)$ is a weight used to enforce the tracking at time instants near to the current time $t$. Combining the cost items (2) and (3) in the target function (1) and minimizing it introduces a trade-off between two competing requirements: the one of charging the PEVs as fast as possible and the one of smoothing the aggregated charging power so that it tracks a target curve which guarantees proper operation of the load area over the time. The connection among the above mentioned competing terms is given by the power balance in the load area and the SOC error dynamics. The former is expressed by the equality constraint

$$
\sum_{r \in R_{t}} p_{r}(\tau / t)=P(\tau / t), \forall \tau \in T_{t}
$$

while the latter is modeled by the linear discrete time invariant system

$e_{r}(\tau+1 / t)=e_{r}(\tau / t)-\left(1-\xi_{r}\right) \Delta t p_{r}(\tau / t), \forall \tau \in T_{t}, \forall r \in R_{t}$ $e_{r}(t / t)=e_{r}(t), \forall r \in R_{t}$

where $\xi_{r}$ is a parameter accounting for the conversion losses, $\Delta t$ represents the sampling time and the intial SOC error $e_{r}(t)$ is the measure taken from the PEV at $t$. In order to enforce the satisfaction of charging preferences, the hard constraints

$$
e_{r}\left(t_{r}^{d e p}\right)=0, \forall r \in R_{t}
$$

is included in the problem formalization, where $t_{r}^{d e p}$ represents the departure time of the $r$-th vehicle.

Finally, other constraints come from the technical limitations on the PEV charging power and the aggregated 
charging power in the load area. We consider in this paper the box contraints

$$
\begin{gathered}
p_{r}^{\text {min }} \leq p_{r}(\tau / t) \leq p_{r}^{\max }, \forall r \in R_{t}, \forall \tau \in T_{t} \\
P^{\text {min }} \leq P(\tau / t) \leq P^{\max }, \forall \tau \in T_{t} .
\end{gathered}
$$

In the light of the above, taking into account control objective 3 and introducing for notational convenience the vectors $p_{r}=\operatorname{col}\left(p_{r}(\tau / t), p_{r}(\tau+1 / t), \ldots, p_{r}(\tau+H-1 / t)\right)$, $P=\operatorname{col}(P(\tau / t), P(\tau+1 / t), \ldots, P(\tau+H-1 / t)), P^{r e f}=$ $\operatorname{col}\left(P^{r e f}(\tau / t), P^{r e f}(\tau+1 / t), \ldots, P^{r e f}(\tau+H-1 / t)\right)$, the open loop optimal control problem at the basis of the MPC scheme can be stated as follow.

Problem 1: For a given set $R_{t}$ of PEVs, each one characterized by a pair of charging preferences $\left(S O C^{r e f}, t^{d e p}\right)$, connected to a load area having an aggregated charging power reference $P^{r e f}$, find a decentralized strategy allowing to compute the optimal values of the control sequences $p_{r}, \forall r \in R_{t}$, and $P$ which minimize the target function (1), such that constraints (4), (5), (6), (7), (8) are satisfied.

\section{Decentralized Solving Procedure}

In this section, we recall the theoretical background of ADMM and we discuss how the open loop optimal control problem can be handled in order to solve it by means of a decentralized approach.

\section{A. Centralized ADMM}

The ADMM is an iterative algorithm which solves optimization problems blending the advantages coming from the decomposability of the dual ascent algorithm and the convergence properties of the method of multipliers [35]. Consider an optimization problem of the form

$$
\begin{array}{cl}
\min _{x, z} & f(x)+g(z) \\
\text { s.t. } & A x+B z=c
\end{array}
$$

where $x \in \mathbb{R}^{n}, z \in \mathbb{R}^{m}, f: \mathbb{R}^{n} \rightarrow \mathbb{R} \cup\{+\infty\}, g: \mathbb{R}^{m} \rightarrow$ $\mathbb{R} \cup\{+\infty\}, A \in \mathbb{R}^{q \times n}, B \in \mathbb{R}^{q \times m}$ and $c \in \mathbb{R}^{q}$. Define the augmented Lagrangian as

$$
L_{\rho}(x, z, \lambda)=L_{0}(x, z, \lambda)+(\rho / 2)\|A x+B z-c\|_{2}^{2}
$$

where $\rho$ is a positive tuning parameter and

$$
L_{0}(x, z, \lambda)=f(x)+g(z)+\lambda^{T}(A x+B z-c)
$$

is the unaugmented Lagrangian related to problem (9). Then consider the following assumptions.

Assumption 1: The functions $f: \mathbb{R}^{n} \rightarrow \mathbb{R} \cup\{+\infty\}$ and $g: \mathbb{R}^{m} \rightarrow \mathbb{R} \cup\{+\infty\}$ are closed, proper, and convex.

Assumption 2: The unaugmented Lagrangian $L_{0}$ has a saddle point.

Assuming assumptions 1 and 2 hold, the ADMM algorithm finds the optimal solution through the following iterations:

$$
\begin{aligned}
x^{k+1} & :=\underset{x}{\arg \min } L_{\rho}\left(x, \lambda^{k}, z^{k}\right) \\
z^{k+1} & :=\underset{z}{\arg \min } L_{\rho}\left(x^{k+1}, \lambda^{k}, z\right) \\
\lambda^{k+1} & :=\lambda^{k}+\rho\left(A x^{k+1}+B z^{k+1}-c\right) .
\end{aligned}
$$

The procedure is iterated until when, for some $k$, the so called primal and dual residuals respectively satisfy the conditions

$$
\begin{array}{r}
\left\|A x^{k+1}+B z^{k+1}-c\right\|_{2}^{2}<\epsilon \\
\left\|\rho A^{T} B\left(z^{k+1}-z^{k}\right)\right\|_{2}^{2}<\epsilon
\end{array}
$$

where the parameter $\epsilon$ is a proper tolerance value. Notice that both $x$-update and $z$-update tasks require the knowledge of $x$ and $z$ at previous or current iteration, so that this procedure can not be executed in parallel by independent agents.

\section{B. Distributed ADMM}

In some cases the ADMM algorithm can be specialized to be executed in a decentralized way. Pose $x=$ $\operatorname{col}\left(x_{1}, x_{2}, \ldots, x_{N}\right)$, where the components $x_{i} \in \mathbb{R}^{n_{i}}$ take the name of local variables, and suppose that the function $f(x)$ can be expressed as a sum of $N$ independent functions $f_{i}\left(x_{i}\right)$. Consider the problem

$$
\begin{array}{ll}
\min _{x, z, \widetilde{z}} & \sum_{i}^{N} f_{i}\left(x_{i}\right)+g(z) \\
\text { s.t. } & x_{i}-\widetilde{z}_{i}=0, \quad i=1 \ldots N
\end{array}
$$

in which the auxiliary variables, components of $\widetilde{z}=$ $\operatorname{col}\left(\widetilde{z}_{1}, \widetilde{z}_{2}, \ldots, \widetilde{z}_{N}\right)$, are introduced to replace the local variables in the linear constraint linking $x$ and $z$. This problem is known as consensus with regularization optimization problem. Considering the augmented Lagrangian of problem (14), the machinery of ADMM brings to the definition of the iteration scheme

$$
\begin{aligned}
x_{i}^{k+1} & :=\underset{x_{i}}{\arg \min } f_{i}\left(x_{i}\right)+\lambda_{i}^{k T} x_{i}+(\rho / 2)\left\|x_{i}-\widetilde{z}_{i}^{k}\right\|_{2}^{2} \\
\left(z^{k+1}, \widetilde{z}^{k+1}\right) & :=\underset{z, \widetilde{z}}{\arg \min } g(z)+\sum_{i=1}^{N}\left(-\lambda_{i}^{k T} \widetilde{z}_{i}+(\rho / 2)\left\|x_{i}^{k+1}-\widetilde{z}_{i}\right\|_{2}^{2}\right) \\
\lambda_{i}^{k+1} & :=\lambda_{i}^{k}+\rho\left(x_{i}^{k+1}-\widetilde{z}_{i}^{k+1}\right)
\end{aligned}
$$

which converges to the optimal solution of (14) under the previously mentioned Assumptions 1 and 2 .

Equations (15) define a decentralized ADMM algorithm able to be executed in parallel by $\mathrm{N}$ local agents each one optimizing a specific local objective $f_{i}\left(x_{i}\right)$, one global agent optimizing the regularization term $g(z)$, driven by the updates of the Lagrangian multipliers $\lambda_{i}$.

\section{Open loop control problem decomposition}

It is straightforward to realize how the machinery of decentralized ADMM can be applied to the open loop optimal control problem defined in section III. Remembering the definition of tracking error and using equation (5), the target function (1) and the power balance constraint (4) can be rewritten in terms of the PEVs charging power and the aggregated charging power to define a problem of the form (9), in which $x=p=\operatorname{col}\left(p_{1}, p_{2}, \ldots, p_{N}\right), N=\left|R_{t}\right|$ and $z=$ $P$. Further the problem (9) is enriched with constraints (6), (7) and (8) affecting separately the behaviour of the PEVs and the load area. Additionally, considering that the target function is separable in the components $p_{r}$, by introducing 
TABLE I: Charging sessions

\begin{tabular}{cccc}
\hline PEV ID & Initial SOC [\%] & Start Time & End Time \\
\hline 1 & $11.46 \%$ & $00: 30$ & $03: 10$ \\
2 & $22.92 \%$ & $00: 30$ & $02: 45$ \\
3 & $26.58 \%$ & $00: 30$ & $02: 50$ \\
4 & $19.25 \%$ & $02: 10$ & $05: 30$ \\
5 & $16.67 \%$ & $02: 10$ & $04: 40$ \\
6 & $19.25 \%$ & $02: 25$ & $05: 05$ \\
7 & $31.67 \%$ & $02: 55$ & $05: 35$ \\
8 & $8.75 \%$ & $04: 25$ & $07: 05$ \\
9 & $30.42 \%$ & $04: 50$ & $08: 10$ \\
10 & $19.25 \%$ & $05: 00$ & $08: 35$ \\
11 & $50,00 \%$ & $06: 25$ & $08: 50$ \\
12 & $35.83 \%$ & $06: 55$ & $09: 30$ \\
13 & $26.58 \%$ & $07: 05$ & $10: 40$ \\
14 & $0,00 \%$ & $07: 55$ & $12: 30$ \\
15 & $8.75 \%$ & $08: 50$ & $11: 20$ \\
16 & $19.25 \%$ & $09: 10$ & $12: 40$ \\
17 & $58.33 \%$ & $09: 35$ & $13: 45$ \\
18 & $8.33 \%$ & $10: 50$ & $13: 25$ \\
19 & $8.33 \%$ & $11: 40$ & $15: 25$ \\
20 & $11.46 \%$ & $12: 30$ & $15: 35$ \\
\hline
\end{tabular}

the auxiliary variables $\widetilde{z}_{i} \in \mathbb{R}^{n_{i}}$, where $n_{i}=H$, the open loop optimal control problem takes the form (14), in which the global variable $z$ is linked to the auxiliary variables through the power balance constraint $z=\sum_{i=1}^{N} \widetilde{z}_{i}$.

In the light of the above, the decentralized ADMM procedure (15) can be applied to the problem at study, provided that the $x_{i}$-update and the $z$-update are performed taking into account the respective additional constraints. Further, notice that the $x_{i}$-update is influenced by local parameters and the auxiliary variable $\widetilde{z}_{i}$, which embeds the behaviour of the other agents, without giving information about them.

\section{Simulation Results}

In order to validate the proposed problem formulation and solving procedure, two simulations have been performed. The control system has been set to work with a sampling time $\Delta t=5$ minutes over a control window of 3 hours (36 sampling periods); the weight of the SOC error cost appearing in target function (1) has been set to $\alpha=0.3$, while the weight of the tracking error cost has been chosen as the function $\beta(\tau / t)=0.6 /(\tau-t+1)$. The augmented Lagrangian parameter and the tolerance of the ADMM algorithm have been set to $\rho=1$ and $\epsilon=0.001$. All the PEVs have a battery capacity $S O C^{\max }=24 \mathrm{kWh}$, conversion losses parameter $\xi=0.1$, minimum and maximum charging power $p^{\text {min }}=$ $0 \mathrm{~kW}$ and $p^{\max }=22 \mathrm{~kW}$. The minimum and maximum aggregated charging power in the load area are $P^{\text {min }}=0 \mathrm{~kW}$ and $P^{\max }=200 \mathrm{~kW}$. The simulations have been performed using Julia 1.0.5 as simulation environment and Gurobi 8.1.1. as optimization solver, running on an Intel®Core ${ }^{\mathrm{TM}} \mathrm{i} 7$ $8565 \mathrm{U}, 1.80 \mathrm{GHz}$ processor. Though the proposed control is decentralized, the simulations are performed on the same processor, in order to assess the computational effort of the control in absence of communications latency among optimization agents.

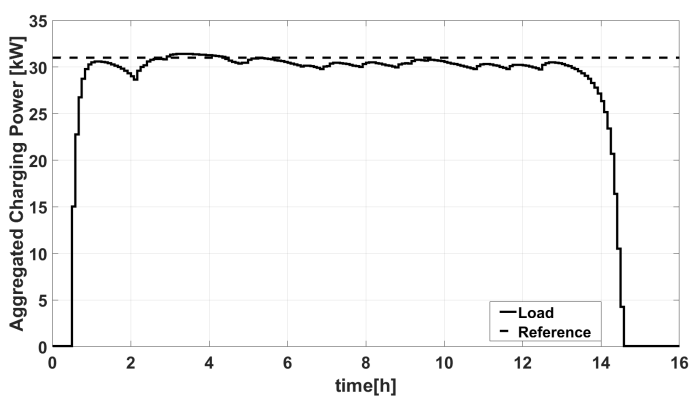

Fig. 1: Sim. I - Aggregated load (continuous line), reference aggregated load (dashed line).

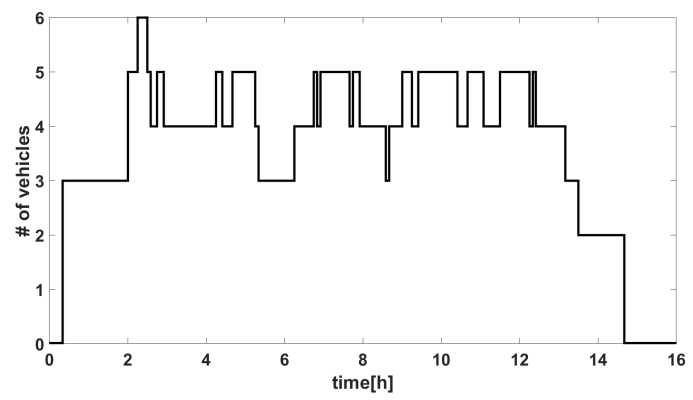

Fig. 2: Sim. I - Number of connected PEVs over the time.

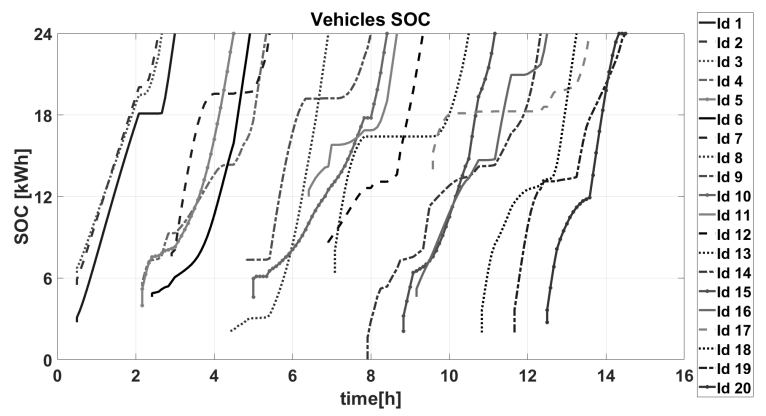

Fig. 3: Sim. I - Evolution of the PEVs state of charge.

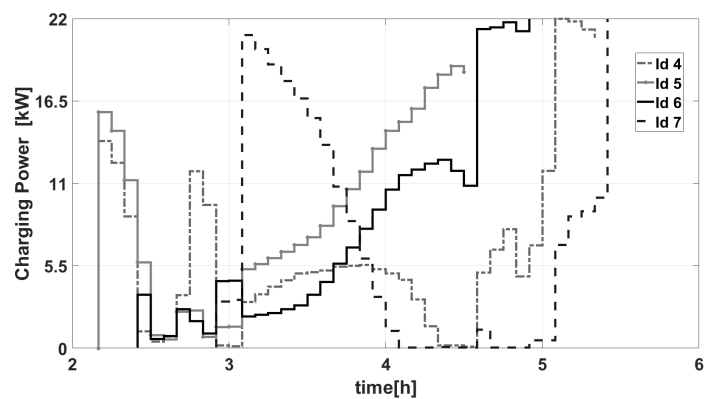

Fig. 4: Sim. I - PEVs charging power, selection of 4 vehicles.

A. $c$

The first simulation is based on the sequence of charging sessions reported in Table I; all the session requests are characterized by a $100 \%$ of desired SOC level. A flat reference power of $31.5 \mathrm{~kW}$ is assigned to the load area. As 


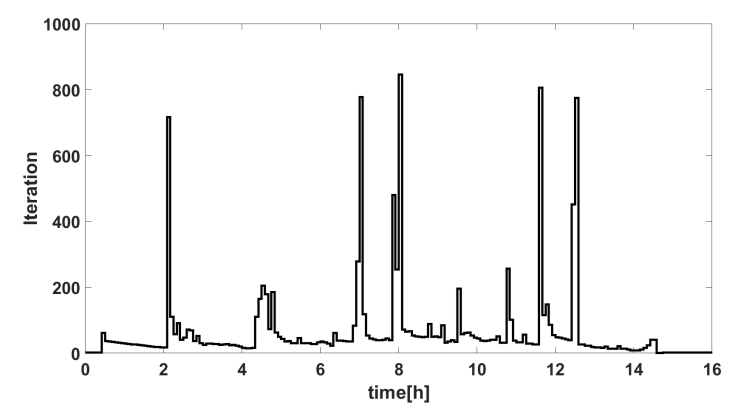

Fig. 5: Sim. I - Number of iterations needed for convergence.

shown in Fig. 1 the proposed decentralized control allows to satisfy the tracking requirement pretty well; once reached the steady-state, the maximum tracking error is $7 \%$ of the reference power. The control properly manages the fluctuations in the number of connected PEVs (Fig. 2), smoothing the aggregated charging power. A careful comparison of Fig. 1 and 2 reveals that degradated tracking performances are expected at times when there is not sufficient request of power in the load area; consequently, the application in a real system requires to properly choose the power reference based on hystorical data, or to properly modify it over the time so that it can be actually tracked by the control system.

Fig. 3 shows the evolution of SOC over the time for all PEVs. All the charging preferences are satisfied in terms of desired SOC at the departure time. Fig. 4 reports the charging power schedules of 4 charging sessions taking place simultaneously. It is interesting to see that, though PEVs do not share information about preferences among them, they collaborate changing their own controls over the time so that the charging preferences of all PEVs in the cluster are met. See for example how PEV 7 changes its schedule at time 03:05 to let PEVs 5 and 6 complete the charging session before their departure time.

Looking at the ADMM performances, Fig. 5 reports the number of iterations needed to find the solution of the open loop optimal control problem at each sampling instant. It is worth noting that large peaks in the number of iterations arise at times when a new charging session becomes part of the optimization problem. Looking at the evolution of the primal and dual residual over the iterations, for the worst case occuring at 08:00 (Fig. 6), it can be noticed that relaxing the tolerance to 0.01 would imply the algorithm to converge approximately within 20 iterations. Finally it is interesting to see how the chosen sampling time affects the convergence performances of the algorithm in terms of needed iterations and related computation time, neglecting the latency of communications among the optimization agents: Fig. 7 related to the first nontrivial optimization occurring at 00:30, provides a realistic indication about the minimum sampling time that can be used in a real time application of the proposed control scheme, and represents a benchmark for assessing the impact of communications latency on the control system performances.
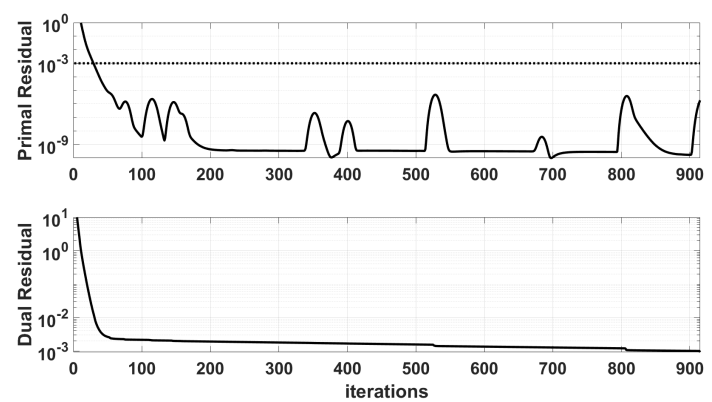

Fig. 6: Sim. I - Evolution of primal and dual residuals for the optimization performed at 08:00.

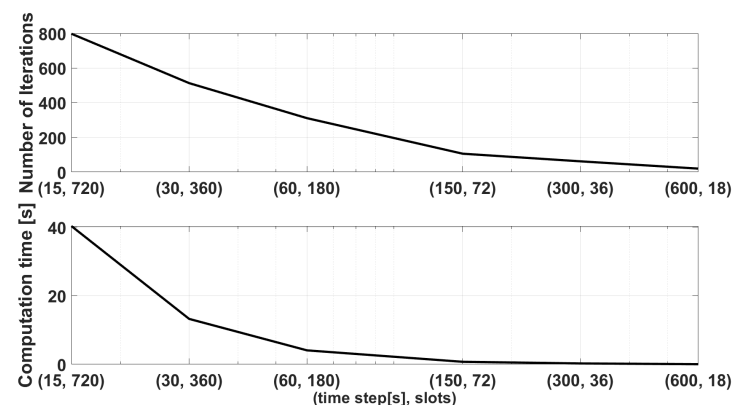

Fig. 7: Sim. I - Number of iterations needed for convergence and computation time versus sampling time, for the optimization performed at 00:30.

\section{B. Simulation II}

The second simulation is aimed at testing the ability of the control system to react to a short-term modification of the load area reference power (DSM signal); examples of application in a real scenario are the provisioning of a power balancing service to the network operator and the reaction to an update in the forecast of power generation from renewable energy sources. To this purpose the same test case characterizing simulation 1 is reconsidered and a DSM signal in sent to the controller notifyng that the reference power is modified starting from 04:00 to gradually relaxing the aggregated charging power to $22 \mathrm{~kW}$ over a 6 hours temporal window. Fig. 8 shows the aggregated schedule which is actually realized at the end of the simulation period: the control system continues to guarantee good tracking performances (9\% of tracking error), while still guaranteeing proper evolution of PEVs' SOC towards the desired SOC.

\section{CONCLUSions And Future Works}

In this paper a MPC based PEVs charging control scheme has been presented. The open loop optimal control problem at the basis of MPC has been modeled as a consesus with regularization optimization problem and solved by means of a decentralized ADMM algorithm. The proposed approach allows to jointly meet control requirements at load area level (reference charging power tracking) and at PEVs level (charging preferences and privacy). The computational effort related to the decentralized optimization algorithm has been 


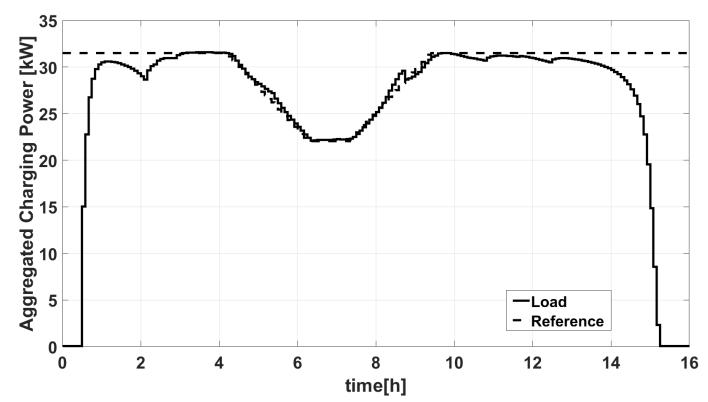

Fig. 8: Sim. II - Aggregated load (continuous line), reference aggregated load (dashed line).

preliminary assessed in terms of number of needed iterations to reach optimality and computation time, assuming zero communication latency among optimization agents. As a result, this study provides a proof about the potential of the proposed control approach and a preliminary evaluation of the computational effort; future works will consider additional requirements (according to the IEC61851 international standard) and the impact assessment of communications reliability and latency on the control system performances, as a result of the design and demonstration activities carried out in the context of the EU 5G Solutions research project.

\section{ACKNOWLEDGMENT}

The authors gratefully acknowledge the CRAT team involved in the 5G-SOLUTIONS project and the other 5GSOLUTIONS consortium members for the fruitful discussions over the paper's matters.

\section{REFERENCES}

[1] European Parliament, Council of the European Union, "Directive 2012/27/EU of the European Parliament and of the Council of 25 October 2012 on energy efficiency, amending Directives 2009/125/EC and 2010/30/EU and repealing Directives 2004/8/EC and 2006/32/EC Text with EEA relevance," OJ, vol. L 315, p. 1-56, 25/10/2012. [Online]. Available: https://eur-lex.europa.eu/legal-content/EN/TXT/ ?uri=OJ:L:2012:315:TOC

[2] European Parliament, Council of the European Union , "Directive (EU) 2018/2001 of the European Parliament and of the Council of 11 December 2018 on the promotion of the use of energy from renewable sources (Text with EEA relevance.)," OJ, vol. L 328, p. 82-209, 11/12/2018. [Online]. Available: https://eur-lex.europa.eu/ legal-content/EN/TXT/?uri=OJ:L:2018:328:TOC

[3] S. Chowdhuri and A. Mukherjee, "Decision making on load management for plug-in hybrid electric vehicle in smart grid," in 2017 IEEE Calcutta Conference (CALCON), Dec 2017, pp. 288-291.

[4] N. Thie, E. Junge, S. Hillenbrand, and M. Konermann, "Evaluation of grid compatible load management concepts for e-mobility in distribution grids," in 2019 54th International Universities Power Engineering Conference (UPEC), Sep. 2019, pp. 1-5.

[5] M. A. Azzouz, M. F. Shaaban, and E. F. El-Saadany, "Real-time optimal voltage regulation for distribution networks incorporating high penetration of pevs," IEEE Transactions on Power Systems, vol. 30, no. 6, pp. 3234-3245, Nov 2015.

[6] C. Wu, H. Mohsenian-Rad, J. Huang, and J. Jatskevich, "Pev-based combined frequency and voltage regulation for smart grid," in 2012 IEEE PES Innovative Smart Grid Technologies (ISGT), Jan 2012, pp. $1-6$.
[7] M. Zeraati, M. E. Hamedani Golshan, and J. M. Guerrero, "A consensus-based cooperative control of pev battery and pv active power curtailment for voltage regulation in distribution networks," IEEE Transactions on Smart Grid, vol. 10, no. 1, pp. 670-680, Jan 2019.

[8] S. Han, H. Aki, and S. Han, "Optimal charging strategy of a pev battery considering frequency regulation and distributed generation," in 2012 IEEE Vehicle Power and Propulsion Conference, Oct 2012, pp. 1014-1019.

[9] K. R. Reddy and S. Meikandasivam, "Load flattening and voltage regulation using plug-in electric vehicle's storage capacity with vehicle prioritization using anfis," IEEE Transactions on Sustainable Energy, vol. 11, no. 1, pp. 260-270, Jan 2020.

[10] S. Khatiri-Doost and M. Amirahmadi, "Peak shaving and power losses minimization by coordination of plug-in electric vehicles charging and discharging in smart grids," in 2017 IEEE International Conference on Environment and Electrical Engineering and 2017 IEEE Industrial and Commercial Power Systems Europe (EEEIC / I CPS Europe), June 2017, pp. 1-5.

[11] S. Pazouki, A. Mohsenzadeh, S. Ardalan, and M. Haghifam, "Simultaneous planning of pev charging stations and dgs considering financial, technical,and environmental effects," Canadian Journal of Electrical and Computer Engineering, vol. 38, no. 3, pp. 238-245, Summer 2015.

[12] R. Yu and Z. Ma, "A distributed charging coordination of plug-in electric vehicles based on potential game considering feeder overload constraint," in 2015 18th International Conference on Electrical Machines and Systems (ICEMS), Oct 2015, pp. 1114-1118.

[13] S. Deb, P. Harsh, J. P. Sahoo, and A. K. Goswami, "Charging coordination of plug-in electric vehicle for congestion management in distribution system integrated with renewable energy sources," in 2018 IEEE International Conference on Power Electronics, Drives and Energy Systems (PEDES), Dec 2018, pp. 1-6.

[14] M. Moghbel, M. A. S. Masoum, and F. Shahnia, "Coordinated charging of pevs in unbalanced residential network based on worst node voltage profile," in 2013 IEEE Power Energy Society General Meeting, July 2013, pp. 1-5.

[15] A. Hamidi, D. Nazarpour, and S. Golshannavaz, "Optimal scheduling of unbalanced distribution networks to improve the contribution of renewables and network balancing performance," in 2017 Iranian Conference on Electrical Engineering (ICEE), May 2017, pp. 12361241.

[16] M. González Vayá and G. Andersson, "Self scheduling of plug-in electric vehicle aggregator to provide balancing services for wind power," IEEE Transactions on Sustainable Energy, vol. 7, no. 2, pp. 886-899, April 2016.

[17] A. G. Fiorese, Y. R. Rodrigues, A. C. Z. de Souza, and M. C. Passaro, "On effects of pevs in islanded microgrids resilience," in 2019 IEEE PES Innovative Smart Grid Technologies Conference - Latin America (ISGT Latin America), Sep. 2019, pp. 1-6.

[18] D. Wu, X. Ke, N. Radhakrishnan, and A. Reiman, "Optimization methods for evaluating pev charging considering customer behavior," in 2018 IEEE Power Energy Society General Meeting (PESGM), Aug 2018, pp. 1-5.

[19] A. Kulvanitchaiyanunt, V. C. P. Chen, J. Rosenberger, P. Sarikprueck, and W. Lee, "A linear program for system-level control of regional phev charging stations," IEEE Transactions on Industry Applications, vol. 52, no. 3, pp. 2046-2052, May 2016

[20] A. Chiş, J. Lundén, and V. Koivunen, "Reinforcement learningbased plug-in electric vehicle charging with forecasted price," IEEE Transactions on Vehicular Technology, vol. 66, no. 5, pp. 3674-3684, May 2017.

[21] K. K. Monfared, H. Iman-Eini, and R. Razi, "Control of single-phase bidirectional pev/ev charger based on fcs-mpc method for v2g reactive power operation," in 2019 10th International Power Electronics, Drive Systems and Technologies Conference (PEDSTC), Feb 2019, pp. 641646.

[22] M. Rahmani-Andebili and M. Fotuhi-Firuzabad, "An adaptive approach for pevs charging management and reconfiguration of electrical distribution system penetrated by renewables," IEEE Transactions on Industrial Informatics, vol. 14, no. 5, pp. 2001-2010, May 2018.

[23] P. Kou, D. Liang, L. Gao, and F. Gao, "Stochastic coordination of plugin electric vehicles and wind turbines in microgrid: A model predictive control approach," IEEE Transactions on Smart Grid, vol. 7, no. 3, pp. 1537-1551, May 2016. 
[24] A. Di Giorgio, F. Liberati, R. Germanà, M. Presciuttini, L. R Celsi, and F. Delli Priscoli, "On the control of energy storage systems for electric vehicles fast charging in service areas," in 2016 24th Mediterranean Conference on Control and Automation (MED). IEEE, Jun. 2016. [Online]. Available: https://doi.org/10.1109/med. 2016.7535947

[25] European Commission. $(2012,2015)$ FP7 - ICT. [Online]. Available: https://cordis.europa.eu/project/id/314328/es

[26] Applied Research to Technologies (Ares2t). (2013). [Online]. Available: https://www.ares2t.com/wordpress/

[27] F. Liberati, A. Mercurio, L. Zuccaro, A. Tortorelli, and A. Di Giorgio, "Electric vehicles charging load reprofiling," in 22nd Mediterranean Conference on Control and Automation, June 2014, pp. 728-733.

[28] S. Boyd, N. Parikh, E. Chu, B. Peleato, and J. Eckstein, "Distributed optimization and statistical learning via the alternating direction method of multipliers," Foundations and Trends in Machine Learning, vol. 3, no. 1, pp. 1-122, 2011. [Online]. Available: http://dx.doi.org/10.1561/2200000016

[29] A. Fiaschetti, V. Suraci, and F. Delli Priscoli, "The SHIELD framework: How to control security, privacy and dependability in complex systems," in 2012 Complexity in Engineering (COMPENG). Proceedings. IEEE, Jun. 2012. [Online]. Available: https://doi.org/ 10.1109/compeng.2012.6242962
[30] A. Di Giorgio, F. Liberati, and S. Canale, "Electric vehicles charging control in a smart grid: A model predictive control approach," Control Engineering Practice, vol. 22, pp. 147 - 162, 2014.

[31] A. Di Giorgio, L. Zuccaro, G. Coppola, and F. Caleno, "Plug-in electric vehicles smart charging in italy: Control system architecture and field test results," in 2018 IEEE International Conference on Environment and Electrical Engineering and 2018 IEEE Industrial and Commercial Power Systems Europe (EEEIC / I CPS Europe), June 2018, pp. 1-5.

[32] A. Di Giorgio, A. Giuseppi, R. Germanà, and F. Liberati, "Decentralised model predictive control of electric vehicles charging," in 2019 IEEE International Conference on Systems, Man, and Cybernetics, SMC 2019, October 2019, pp. 1-7.

[33] 5G-SOLUTIONS Consortium. (2019) H2020. [Online]. Available: https://www.5gsolutionsproject.eu/

[34] D. Mayne, J. Rawlings, C. Rao, and P. Scokaert, "Constrained model predictive control: Stability and optimality," Automatica, vol. 36, no. 6, pp. 789 - 814, 2000. [Online]. Available: http://www.sciencedirect.com/science/article/pii/S0005109899002149

[35] S. Boyd, N. Parikh, E. Chu, B. Peleato, J. Eckstein et al., "Distributed optimization and statistical learning via the alternating direction method of multipliers," Foundations and Trends ${ }^{\circledR}$ in Machine learning, vol. 3, no. 1, pp. 1-122, 2011. 